\title{
CHANNEL IDENTIFICATION UNDER DOPPLER AND TIME SHIFTS USING MIXED TRAINING SIGNALS
}

\author{
XIANG-GEN XIA \\ Communicated by Charles Groetsch \\ This paper is dedicated with affection to Professor Zuhair Nashed.

\begin{abstract}
Channel identification in the presence of Doppler is not as well studied as the one free from Doppler due to the difficulty caused from the time-varying characteristics of the channel. In this paper, we present a method to identify channels with both Doppler and time shifts using mixed training signals. The training signals we use consist of two parts, where one part is a constant and the other part is a conventional training signal, such as a pseudo-random signal or a chirp signal. These two parts in a training signal may be separated either in the time domain or in the frequency domain. The constant signal part is used to identify the Doppler shifts and the other part is used to identify the time shifts. We provide a necessary and sufficient condition on the channel identifiability in terms of the time and Doppler shifts when mixed training signals are used. It can be shown that the condition holds almost surely in most cases of interest in practice.
\end{abstract}

1. Introduction. Doppler and time shifts (or delays or spread) usually occur in wireless mobile communication systems with high speed transmission, which often causes problems of channel impairments. Due to the Doppler shifts of moving vehicles, the channel is usually modeled as a time variant linear system and is not as well studied as a timeinvariant linear channel is. There has been a tremendous amount of research on time-invariant linear system identification with both blind

This work was supported in part by the Air Force Office of Scientific Research (AFOSR) under Grant No. FA 9550-08-1-0219 and the National Science Foundation under Grant CCR-0325180.

Received by the editors on January 25, 2007, and in revised form on April 9, 2007.

DOI:10.1216/JIE-2008-20-3-393 Copyright (c)2008 Rocky Mountain Mathematics Consortium 
and non-blind (using training signals) methods ${ }^{1}$ This is, however, not equally the case for time-variant linear system identification. Some researches on this topic have appeared, such as [1-10], and increasing attention has being paid mainly because of the need of wireless high speed data communications.

In this paper, we focus on the problem of the channel identification in the presence of both Doppler and time shifts by using training signals. Specifically, the following channel model studied in [1] is used. Let $x(t)$ and $y(t)$ be transmitted and received signals, respectively. Then

$$
y(t)=\sum_{k=1}^{N_{p}} \alpha_{k} x\left(t-\tau_{k}\right) e^{j \omega_{k} t}+n(t),
$$

where $\alpha_{k}, \tau_{k}$, and $\omega_{k}$ are the path coefficient (complex-valued), the time shift (real-valued), and the Doppler shift (real-valued) of the $k$ th multipath component in the channel, respectively, and $N_{p}$ is the number of the total multipath components, and $n(t)$ is the channel additive noise. The Doppler shifts $\omega_{k} \approx 2 v \omega_{c} / c$ with the carrier frequency $\omega_{c}$, the velocity $v$ of the moving object, and the velocity $c$ of light. The channel identification here is to estimate the unknown parameters $\left\{\left(\alpha_{k}, \tau_{k}, \omega_{k}\right), 1 \leq k \leq N_{p}\right\}$ through knowledge of the transmitted and the received signals $x(t)$ and $y(t)$ in (1.1). Most of the existing methods for the channel identification are based on single type training signals called pilot signals.

In this paper, we propose to use mixed training signals in the above channel identification, which have two parts separated either in the time domain or in the frequency domain. One part of the training signal is a constant and the other part is a pseudo-random signal or other type of linear time-invariant (LTI) channel identification training signals, such as chirps. The constant part is used to identify the Doppler shifts $\omega_{k}$ and the other part is used to identify the time shifts $\tau_{k}$. The corresponding multipath coefficients $\alpha_{k}$ are identified using both parts. Note that not all channels in (1.1) can be identified with this approach. A necessary and sufficient condition in terms of the Doppler and time shifts on the channel identifiability is given. It turns out that almost all channels (1.1) are identifiable with the approach proposed in this paper in most cases of practical interest.

\footnotetext{
1 Since these methods are not used in this paper, they are not cited here.
} 
This paper is organized as follows. In Section 2, we present channel analysis and mixed training signal analysis. In Section 3, we present a necessary and sufficient condition for the identifiability. In Section 4, numerical simulations are presented.

2. Channel and Mixed Training Signal Analyses. Let us first analyze the received signal $y(t)$ in (1.1). To analyze the identifiability, for convenience we assume the additive noise $n(t)$ in the model (1.1) does not appear, i.e., $n(t)=0$. Suppose the transmitted training signal $x(t)$ is a constant, say 1 . Then (1.1) becomes

$$
y(t)=\sum_{k=1}^{N_{p}} \alpha_{k} e^{j \omega_{k} t} .
$$

If all Doppler shifts $\omega_{k}, k=1,2, \ldots, N_{p}$, are distinct, then, by taking a discrete Fourier transform of a certain length for a segment of the received signal $y(t)$, all Doppler shifts $\omega_{k}$ and multipath coefficients $\alpha_{k}$ may be detected. If there are duplications of the Doppler shifts $\omega_{k}$, all the (distinct) Doppler shifts can still be detected with the method above, but not all the coefficients $\alpha_{k}$. For instance, assume $\omega_{1}=\omega_{2}$ and it is not equal to other $\omega_{k}$. Equation (2.1) becomes

$$
y(t)=\left(\alpha_{1}+\alpha_{2}\right) e^{j \omega_{1} t}+\sum_{k=3}^{N_{p}} \alpha_{k} e^{j \omega_{k} t} .
$$

In this case, only the sum $\alpha_{1}+\alpha_{2}$ of the two coefficients $\alpha_{1}$ and $\alpha_{2}$ can be detected, which is not enough to detect their individual values $\alpha_{1}$ and $\alpha_{2}$. However, the Doppler frequencies $\left\{\omega_{1}, \omega_{2}, \ldots, \omega_{N_{p}}\right\}$ are still detectable.

Similarly the time shifts $\tau_{k}$ can be detected in the frequency domain of (1.1) as follows. Taking the Fourier transform of (1.1) we have

$$
Y\left(e^{j \omega}\right)=\sum_{k=1}^{N_{p}} \alpha_{k} X\left(\omega-\omega_{k}\right) e^{-i \tau_{k}\left(\omega-\omega_{k}\right)},
$$

where $Y\left(e^{j \omega}\right)$ and $X\left(e^{j \omega}\right)$ are the Fourier transforms of $y(t)$ and $x(t)$, respectively. Suppose $X\left(e^{j \omega}\right)$ is a constant, say 1 . Then 


$$
Y\left(e^{j \omega}\right)=\sum_{k=1}^{N_{p}} \alpha_{k} e^{-i \tau_{k}\left(\omega-\omega_{k}\right)} .
$$

If all time shifts $\tau_{k}, k=1,2, \ldots, N_{p}$, are distinct, all these time shifts $\tau_{k}$ and the coefficients $\alpha_{k}$ can be detected by taking an inverse discrete Fourier transform of (2.4). Similar to the previous time domain analysis, it is not possible to detect all the coefficients $\alpha_{k}$ when not all the time shifts $\tau_{k}$ are distinct. Consider a training signal $x(t)$ that has two parts either separated in the time domain or in the frequency domain.

When $x(t)$ has two parts separated in the time domain, it has the following form:

$$
x(t)= \begin{cases}x_{0}, & T_{0}<t<T_{1}, \\ x_{1}(t), & T_{1}<t<T_{2},\end{cases}
$$

where $x_{0}$ is a nonzero constant and $x_{1}(t)$ is a conventional pseudorandom signal or the delta pulse, i.e., its Fourier transform $X_{1}\left(e^{j \omega}\right)$ is a constant (flat). In the detection, these two parts are processed separately.

When $x(t)$ has two parts separated in the frequency domain, it has the following form:

$$
x(t)=x_{0}+x_{1}(t) e^{j \omega_{0} t},
$$

where $x_{0}$ and $x_{1}(t)$ are as in (2.5) and $\omega_{0}$ is a frequency shift. In this case, the received signal $y(t)$ is first filtered by using a lowpass filter to extract the constant part $x_{0}$ and a bandpass filter to extract $x_{1}(t)$. We, however, do not want to filter out other information in $y(t)$. Therefore, the frequency $\omega_{0}$ needs to be at least greater than all the Doppler shifts $\omega_{k}$, i.e.

$$
\left|\omega_{0}\right|>\left|\omega_{k}\right|, \quad k=1,2, \ldots, N_{p} .
$$

In what follows, for convenience we use the training signal in (2.5), i.e., the two parts are separated in the time domain. Without loss of 
generality, for simplicity we assume that the two part information is available at the same time interval, for example, $[0, T]$, and

$$
y_{1}(t)=\sum_{k=1}^{N_{p}} \alpha_{k} e^{j \omega_{k} t} x_{0}, \quad t \in[0, T],
$$

and

$$
y_{2}(t)=\sum_{k=1}^{N_{p}} \alpha_{k} e^{j \omega_{k} t} x_{1}\left(t-\tau_{k}\right), \quad t \in[0, T] .
$$

The goal here is to identify the unknown parameters $\left\{\left(\alpha_{k}, \tau_{k}, \omega_{k}\right), 1 \leq\right.$ $\left.k \leq N_{p}\right\}$ from the above equations (2.8) and (2.9). In the following, we also assume that the sampling interval length of the received signals $y_{1}(t)$ and $y_{2}(t)$ is small enough so that all the distinct Doppler shifts $\omega_{k}$ in (2.1) and the distinct time shifts $\tau_{k}$ in (2.4) can be detected by using the discrete Fourier transform (DFT) and the inverse discrete Fourier transform (IDFT) as discussed above. Since these two sets of distinct Doppler shifts and time shifts are obtained from two different DFT and IDFT, their orders and their corresponding coefficients $\alpha_{k}$ may be different, which may cause an identifiability problem. To study this problem, we first have the following straightforward result.

Theorem 1. Let $x(t)$ be a training signal with the two parts as described above. If either all the Doppler shifts $\omega_{k}, 1 \leq k \leq N_{p}$, or all the time shifts $\tau_{k}, 1 \leq k \leq N_{p}$, are distinct, then the unknown parameters $\left\{\left(\alpha_{k}, \tau_{k}, \omega_{k}\right), 1 \leq k \leq N_{p}\right\}$ are detectable by applying the DFT in the time domain and the IDFT in the frequency domain to the two parts of the received data corresponding to the two parts of the training signal, respectively.

Proof: Without loss of generality, we assume $\omega_{k}, 1 \leq k \leq N_{p}$, are distinct. Thus, $\alpha_{k}, \omega_{k}, 1 \leq k \leq N_{p}$, can be detected with the correct order. Since $\tau_{k}, 1 \leq k \leq N_{p}$, may have repetitions, only the linear combinations of $\alpha_{k}$ from the IDFT of (2.9) and the corresponding $\tau_{k}$ can be detected. Form the detected $\alpha_{k}, 1 \leq k \leq N_{p}$, from (2.8), the corresponding $\tau_{k}$ with the same order of $\alpha_{k}$ and $\omega_{k}$ can be determined by checking the linear combinations of the detected $\alpha_{k}$. 
In the following section, we want to present a necessary and sufficient condition on the identifiability of $\left\{\alpha_{k}, \omega_{k}, \tau_{k}\right\}$.

\section{Necessary and Sufficient Condition on the Identifiability.}

In this section, we first present a necessary and sufficient condition and then study the probability that the condition holds.

3.1 Necessary and Sufficient Condition. Since the Doppler shifts $\omega_{k}, 1 \leq k \leq N_{p}$, and the time shifts $\tau_{k}, 1 \leq k \leq N_{p}$, only take finite possible values, such as in $\mathrm{Hz}$ and $\mu$ s, respectively, they may have repetitions. In other words, $\omega_{k_{1}}$ (or $\tau_{k_{1}}$ ) may be equal to $\omega_{k_{2}}$ (or $\tau_{k_{2}}$ ) for $k_{1} \neq k_{2}$. Therefore the identifiability problem now arises from the possible duplications of the Doppler shifts $\omega_{k}$ and the time shifts $\tau_{k}$ as discussed in (2.2).

Although individuals of $\omega_{k}$ and $\tau_{k}$ may have repetitions, the pairs $\left(\omega_{k}, \tau_{k}\right), 1 \leq k \leq N_{p}$, do not have a repetition and otherwise the duplicated pairs (or multiple paths) may be grouped together into a single term (path) in (1.1). Therefore, in what follows we always assume that all the pairs $\left(\omega_{k}, \tau_{k}\right), 1 \leq k \leq N_{p}$, are distinct, i.e., if $\omega_{k_{1}}=\omega_{k_{2}}$, then $\tau_{k_{1}} \neq \tau_{k_{2}}$; and if $\tau_{k_{1}}=\tau_{k_{2}}$, then $\omega_{k_{1}} \neq \omega_{k_{2}}$.

A general setting of the repetitions of $\omega_{k}$ and $\tau_{k}$ is as follows. Let $I_{1}, \ldots, I_{f}$ be a partition of the integer set

$$
\mathcal{I} \triangleq\left\{1,2, \ldots, N_{p}\right\}
$$

such that all the Doppler shifts $\omega_{k}$ for $k \in I_{l}$ for any fixed $l$ are equal, i.e.,

$$
\omega_{k}=\tilde{\omega}_{l} \quad \text { for all } \quad k \in I_{l},
$$

but $\omega_{k}$ in different $I_{l}$ are different, where "partition" means any two sets $I_{l_{1}}$ and $I_{l_{2}}$ for $l_{1} \neq l_{2}$ do not intersect, i.e., $I_{l_{1}} \cap I_{l_{2}}=\phi$ for $l_{1} \neq l_{2}$, and the union of all $I_{l}$ is the integer set $\mathcal{I}$, i.e.,

$$
\bigcup_{l=1}^{f} I_{l}=\mathcal{I},
$$


and each set $I_{l}$ is not empty. Let $J_{1}, \ldots, J_{g}$ be another partition of the integer set $\mathcal{I}$ such that all the time shifts $\tau_{k}$ for $k \in I_{l}$ for any fixed $l$ are equal, i.e.,

$$
\tau_{k}=\tilde{\tau}_{l} \quad \text { for all } \quad k \in J_{l} .
$$

We use $|S|$ to denote the cardinality of a set $S$. Since all pairs $\left(\omega_{k}, \tau_{k}\right)$, $k=1,2, \ldots, N_{p}$, are distinct as assumed before, the intersection of any set $I_{l_{1}}$ and any set $J_{l_{2}}$ has at most one element, i.e.,

$$
\left|I_{l_{1}} \cap J_{l_{2}}\right| \leq 1,
$$

but $\tau_{k}$ in different $J_{l}$ are different,

Similar to the discussion in (2.2), the following summations can only be detected from the DFT of $y_{1}(t)$ in (2.8) and the IDFT of the Fourier transform $Y_{2}\left(e^{j \omega}\right)$ of $y_{2}(t)$ in $(2.9)$ :

$$
\begin{aligned}
& \sum_{k \in I_{l}} \alpha_{k}=\beta_{l}, \quad 1 \leq l \leq f, \\
& \sum_{k \in J_{l}} \alpha_{k}=\gamma_{l}, \quad 1 \leq l \leq g,
\end{aligned}
$$

where $\beta_{l}$ and $\gamma_{l}$ are the detected values, and the two partitions $\left\{I_{l_{1}}\right\}$ and $\left\{J_{l_{2}}\right\}$ are not known. The identifiability of $\left\{\left(\alpha_{k}, \tau_{k}, \omega_{k}\right), 1 \leq k \leq N_{p}\right\}$ is then reduced to the solvability of these equations (3.4)-(3.5) with unknown partitions $\left\{I_{l_{1}}\right\}$ and $\left\{J_{l_{2}}\right\}$. The following is a necessary and sufficient condition for the identifiability.

Theorem 2. The parameters $\left\{\left(\alpha_{k}, \tau_{k}, \omega_{k}\right), 1 \leq k \leq N_{p}\right\}$ are uniquely determined using the above mixed training signal model if and only if there does not exist any path $k, 1 \leq k \leq N_{p}$, such that both the $k$ th Doppler shift $\omega_{k}$ and the $k$ th time shift $\tau_{k}$ have their repetitions.

A necessary and sufficient condition in Theorem 2 can be stated in the following mathematical way: there do not exist three distinct integers $k$, $k_{1}$, and $k_{2}$ such that $\omega_{k}=\omega_{k_{1}}$ and $\tau_{k}=\tau_{k_{2}}$. The proof of the necessary part of Theorem 2 is in the Appendix. To prove the sufficiency part, we need the following result. 
Lemma 1. There does not exist any path $k, 1 \leq k \leq N_{p}$, such that both the kth Doppler shift $\omega_{k}$ and the kth time shift $\tau_{k}$ have their repetitions if and only if there are two subsets $I$ and $J$ of $\left\{1,2, \ldots, N_{p}\right\}$ such that their union is $\mathcal{I}$, that is, $I \cup J=\mathcal{I}=\left\{1,2, \ldots, N_{p}\right\}$, and all the Doppler shifts $\omega_{k}$ for $k \in I$ are distinct in the set of all $\omega_{k}$ for $k \in\left\{1,2, \ldots, N_{p}\right\}$ and all the time shifts $\tau_{k}$ for $k \in J$ are distinct in the set of all $\tau_{k}$ for $k \in\left\{1,2, \ldots, N_{p}\right\}$.

Proof: We first prove the necessity part. Let $F=\left\{l_{1}: 1 \leq\right.$ $l_{1} \leq f$ and $\left.\left|I_{l_{1}}\right|>1\right\}$ and $I_{c}=\cup_{l_{1} \in F} I_{l_{1}}$ and $G=\left\{l_{2}: 1 \leq l_{2} \leq\right.$ $g$ and $\left.\left|J_{l_{2}}\right|>1\right\}$ and $J_{c}=\cup_{l_{2} \in G} J_{l_{2}}$. By the condition, $I_{c}$ and $J_{c}$ don't intersect, i.e., $I_{c} \cap J_{c}=\phi$. Let $I$ and $J$ be the complementary sets of $I_{c}$ and $J_{c}$ of $\left\{1,2, \ldots, N_{p}\right\}$, respectively. Since all $\omega_{k}$, for $k \in I$, and all $\tau_{k}$, for $k \in J$, are distinct, by $I_{c} \cap J_{c}=\phi$ we have $J_{c} \subset I$ and $I_{c} \subset J$. Therefore, $I \cup J=\left\{1,2, \ldots, N_{p}\right\}$.

We now prove the sufficiency part. Assume that there exist three distinct integers $k, k_{1}$, and $k_{2}$ such that $\omega_{k}=\omega_{k_{1}}$ and $\tau_{k}=\tau_{k_{2}}$. Then, the integer $k$ will not be in any integer sets $I$ and $J$ as described in the lemma, i.e., $I \cup J \neq\left\{1,2, \ldots, N_{p}\right\}$.

Lemma 2. If there are two subsets $I$ and $J$ of $\left\{1,2, \ldots, N_{p}\right\}$ such that their union is $I \cup J=\left\{1,2, \ldots, N_{p}\right\}$, and all the Doppler shifts $\omega_{k}$ for $k \in I$ are distinct in the set of all $\omega_{k}$ for $k \in\left\{1,2, \ldots, N_{p}\right\}$ and all the time shifts $\tau_{k}$ for $k \in J$ are distinct in the set of all $\tau_{k}$ for $k \in\left\{1,2, \ldots, N_{p}\right\}$, then $\left\{\alpha_{k}, \omega_{k}, \tau_{k}\right\}, k \in\left\{1,2, \ldots, N_{p}\right\}$, can be uniquely determined.

Proof: Two sets of coefficients $\beta_{k}$ from (2.8) and the DFT and $\gamma_{k}$ from (2.9) and the IDFT can be solved. By the condition in the lemma, it is known that $\alpha_{k}=\beta_{k}$ for $k \in I$ and $\alpha_{k}=\gamma_{k}$ for $k \in J$. Since $I \cup J=\left\{1,2, \ldots, N_{p}\right\}$, all the $\alpha_{k}$ can be detected. The rest is the same as the proof of Theorem 1 by checking the linear combinations of $\alpha_{k}$ for the order determinations of $\omega_{k}$ and $\tau_{k}$.

The sufficiency part in Theorem 2 is a consequence of Lemma 1 and Lemma 2. From Theorem 2 and Lemma 1, it is immediate that the condition in Lemma 2 is also necessary, i.e., the following corollary 
holds.

Corollary 1. The parameters $\left\{\alpha_{k}, \omega_{k}, \tau_{k}\right\}, k \in\left\{1,2, \ldots, N_{p}\right\}$, can be uniquely determined using the mixed training signal model if and only if there are two subsets $I$ and $J$ of $\left\{1,2, \ldots, N_{p}\right\}$ such that their union is $I \cup J=\left\{1,2, \ldots, N_{p}\right\}$, and all the Doppler shifts $\omega_{k}$ for $k \in I$ are distinct in the set of all $\omega_{k}$ for $k \in\left\{1,2, \ldots, N_{p}\right\}$ and all the time shifts $\tau_{k}$ for $k \in J$ are distinct in the set of all $\tau_{k}$ for $k \in\left\{1,2, \ldots, N_{p}\right\}$.

3.2 Probability Analysis. In the following, we access the probability of channel identifiability in the case of four paths, i.e., for the conditions in Theorems 1-2 to hold, when $N_{p}=4$. The condition is in terms of the Doppler and time shifts $\omega_{k}$ and $\tau_{k}$. Since in practical digital processing, these Doppler and time shifts are quantized to finite values. For convenience, we assume that there are total $M_{d}$ possible different values of the Doppler shifts and total $M_{t}$ possible values for the time shifts. In other words, each $\omega_{k}$ may take one of $M_{d}$ different values

$$
D_{\text {range }}=\left\{v_{d, 1}, v_{d, 2}, \ldots, v_{d, M_{d}}\right\},
$$

and each $\tau_{k}$ may take one of $M_{t}$ different values

$$
T_{\text {range }}=\left\{v_{t, 1}, v_{t, 2}, \ldots, v_{t, M_{t}}\right\} .
$$

For example, $D_{\text {range }}=\{-50 \mathrm{~Hz},-49 \mathrm{~Hz}, \ldots, 50 \mathrm{~Hz}\}$ and $T_{\text {range }}=$ $\{0 \mu \mathrm{s}, 1 \mu \mathrm{s}, \ldots, 100 \mu \mathrm{s}\}$. The two numbers $M_{d}$ and $M_{t}$ can be determined when the Doppler spread width $f_{m}$ and the rms time spread width $\sigma_{\tau}$ are known for a given channel.

As we mentioned earlier, we have a sufficient condition in Theorem 1 and a necessary and sufficient condition in Theorem 2 . These two conditions coincide for $N_{p}=1,2,3$. Although the probability expressions for the conditions in Theorems 1-2 to hold for a general $N_{p}$ are complicated, in the following we calculate them when $N_{p}=4$.

Clearly, the total number of all distinct $N_{p}$ pairs of $\left(\omega_{k}, \tau_{k}\right)$ is

$$
P_{\text {total }}=\left(\begin{array}{c}
M_{d} M_{t} \\
N_{p}
\end{array}\right) .
$$


We first consider the condition in Theorem 1, i.e., either all $\omega_{k}$ for $1 \leq k \leq N_{p}$ or all $\tau_{k}$ for $1 \leq k \leq N_{p}$ are distinct. By some calculations, it is not hard to see that the total number of such $N_{p}$ pairs $\left(\omega_{k}, \tau_{k}\right)$ is

$$
\begin{aligned}
P_{1}= & \left(\begin{array}{c}
M_{d} \\
4
\end{array}\right)\left(\begin{array}{c}
M_{t} \\
1
\end{array}\right)+\left(\begin{array}{c}
M_{d} \\
4
\end{array}\right)\left(\begin{array}{c}
M_{t} \\
2
\end{array}\right)\left[\frac{4 !}{1 ! 3 !}+\frac{4 !}{2 ! 2 !}+\frac{4 !}{1 ! 3 !}\right] \\
& +\left(\begin{array}{c}
M_{d} \\
4
\end{array}\right)\left(\begin{array}{c}
M_{t} \\
3
\end{array}\right)\left(\begin{array}{l}
3 \\
1
\end{array}\right) \frac{4 !}{1 ! 1 ! 2 !} \\
& +\left(\begin{array}{c}
M_{t} \\
4
\end{array}\right)\left(\begin{array}{c}
M_{d} \\
1
\end{array}\right)+\left(\begin{array}{c}
M_{t} \\
4
\end{array}\right)\left(\begin{array}{c}
M_{d} \\
2
\end{array}\right)\left[\frac{4 !}{1 ! 3 !}+\frac{4 !}{2 ! 2 !}+\frac{4 !}{1 ! 3 !}\right] \\
& +\left(\begin{array}{c}
M_{t} \\
4
\end{array}\right)\left(\begin{array}{c}
M_{d} \\
3
\end{array}\right)\left(\begin{array}{l}
3 \\
1
\end{array}\right) \frac{4 !}{1 ! 1 ! 2 !}+\left(\begin{array}{c}
M_{t} \\
4
\end{array}\right)\left(\begin{array}{c}
M_{d} \\
4
\end{array}\right) 4 ! .
\end{aligned}
$$

Therefore, the probability is

$$
\begin{aligned}
& \text { Probability (either all } \omega_{k} \text { for } 1 \leq k \leq N_{p} \text { or all } \tau_{k} \\
& \qquad \text { for } 1 \leq k \leq N_{p} \text { are distinct) }=\frac{P_{1}}{P_{\text {total }}}
\end{aligned}
$$

where $P_{\text {total }}$ is defined in (3.8) with $N_{p}=4$ and $P_{1}$ is defined in (3.9).

The total number of pairs $\left(\omega_{k}, \tau_{k}\right)$ that satisfy the necessary and sufficient condition in Theorem 2 is

$$
\begin{aligned}
P_{2}=\left(\begin{array}{c}
M_{d} \\
4
\end{array}\right) M_{t}^{4}+\left(\begin{array}{c}
M_{d} \\
3
\end{array}\right)\left(\begin{array}{l}
3 \\
1
\end{array}\right)\left(\begin{array}{c}
M_{t} \\
2
\end{array}\right) M_{t}^{2} \\
+\left(\begin{array}{c}
M_{d} \\
2
\end{array}\right)\left[\left(\begin{array}{c}
M_{t} \\
2
\end{array}\right)\left(\begin{array}{c}
M_{t}-2 \\
2
\end{array}\right)+\left(\begin{array}{c}
2 \\
1
\end{array}\right)\left(\begin{array}{c}
M_{t} \\
3
\end{array}\right) M_{t}\right] .
\end{aligned}
$$

Therefore, the probability for the condition in Theorem 2 is

$$
\begin{aligned}
& \text { Probability (the necessary and sufficient } \\
& \qquad \text { condition in Theorem } 2 \text { holds })=\frac{P_{2}}{P_{\text {total }}},
\end{aligned}
$$

where $P_{\text {total }}$ is defined in (4.3) with $N_{p}=4$ and $P_{2}$ is defined in (4.6). 
To compare the above two probabilities, their corresponding curves are plotted in Fig. 1, where we set $M_{d}=M_{t}$ and the x-axis indicates the variable $M_{d}$, which is from 4 to 101 . One can clearly see that the probability for the necessary and sufficient condition in Theorem 2 is above the one for sufficient condition in Theorem 1. When the total numbers $M_{d}$ and $M_{t}$ of the possible Doppler and time shifts are large relative to the total number $N_{p}$ of multipath components in a channel, the necessary and sufficient condition in Theorem 2 holds almost surely, i.e., the probability is very close to 1 .

4. Numerical Simulations. In the following simulations, we use $N_{p}=4$, and

$$
\frac{\omega_{k}}{2 \pi} \in D_{\text {range }}=\{-50 \mathrm{~Hz},-49 \mathrm{~Hz}, \ldots, 50 \mathrm{~Hz}\}, \quad k=1,2,3,4,
$$

and

$$
\tau_{k} \in T_{\text {range }}=\{0 \mu \mathrm{s}, 1 \mu \mathrm{s}, \ldots, 100 \mu \mathrm{s}\}, \quad k=1,2,3,4 .
$$

The Doppler and time shifts $\omega_{k}$ and $\tau_{k}$ for $k=1,2,3,4$ are randomly chosen from the above sets $D_{\text {range }}$ and $T_{\text {range }}$, respectively, such that all pairs $\left(\omega_{k}, \tau_{k}\right)$ for $k=1,2,3,4$ are distinct. The multipath coefficients $\alpha_{k}$ for $k=1,2,3,4$ are randomly chosen from Gaussian random processes with all possible real values.

For the first piece $y_{1}(t)$ of data, the sampling rate is chosen as $1 / T=128$, i.e.,

$$
y_{1}[l]=y_{1}(l / 128)=\sum_{k=1}^{N_{p}} \alpha_{k} e^{j l \omega_{k} / 128}+n_{1}(l / 128), \quad-63 \leq l \leq 64 .
$$

For the second piece $Y_{2}(\omega)$ of data, the sampling rate is chosen $1 / T=128 /(2 \pi)$, i.e.,

$$
\begin{aligned}
& Y_{2}[l]=Y_{2}(2 \pi l / 128)=\sum_{k=1}^{N_{p}} \alpha_{k} e^{j l 2 \pi \tau_{k} / 128}+n_{2}(2 \pi l / 128), \\
& 0 \leq l \leq 127 \text {. }
\end{aligned}
$$


Fig. 2 shows the curves of the ratios of the mean square errors (MSE) of the true $\omega_{k}, \tau_{k}$, and $\alpha_{k}$, and their detected values over their mean powers. The $\mathrm{x}$-axis is the ratios of the mean powers of the multipath coefficients $\alpha_{k}$ over the variance of the additive noise $n_{i}, i=1,2$, in (4.1-4.2). In Fig. 2, 10000 Monte Carlo simulations are implemented.

5. Conclusions. In this paper, we proposed a channel identification algorithm using a mixed training signal, where the channel has both the Doppler shifts and time shifts. The mixed training signals consist of two parts with one part constant and the other part a conventional training signal, such as a pseudo-random signal. These two parts of the signals may be separated either in the time domain or in the frequency domain. The constant part can be used to detect the Doppler shifts while the other part can be used to detect the time shifts. Both parts are used to detect the corresponding coefficients and the synchronization between the detected Doppler and time shifts. A necessary and sufficient condition was given for the channel identifiability based on the mixed training signal approach. A probability analysis for the identifiability was presented. It turns out that almost all channels of practical interests are identifiable. Finally, some simple numerical examples were presented.

Appendix: The Necessity Proof of Theorem 2 The linear equations in $(3.4-3.5)$ can be expressed by the following matrix form:

$$
A\left[\begin{array}{c}
\alpha_{1} \\
\vdots \\
\alpha_{N_{p}}
\end{array}\right]=\left[\begin{array}{c}
\beta_{1} \\
\vdots \\
\beta_{f} \\
\gamma_{1} \\
\vdots \\
\gamma_{g}
\end{array}\right]
$$

where $A$ is an $f+g$ by $N_{p}$ matrix with only 0 and 1 entries as follows 


$$
A=\left[\begin{array}{c}
\mathbf{a}_{1} \\
\vdots \\
\mathbf{a}_{f} \\
\mathbf{b}_{1} \\
\vdots \\
\mathbf{b}_{g}
\end{array}\right],
$$

where $\mathbf{a}_{l}=\left(a_{l}(k)\right)_{1 \leq k \leq N_{p}}, 1 \leq l \leq f$, with

$$
a_{l}(k)=\left\{\begin{array}{l}
1, \text { if } k \in I_{l}, \\
0, \text { otherwise }
\end{array}\right.
$$

$\mathbf{b}_{l}=\left(b_{l}(k)\right)_{1 \leq k \leq N_{p}}, 1 \leq l \leq g$, with

$$
b_{l}(k)=\left\{\begin{array}{l}
1, \text { if } k \in J_{l}, \\
0, \text { otherwise }
\end{array}\right.
$$

where $\left|I_{l_{1}} \cap I_{l_{2}}\right| \leq 1$ for $1 \leq l_{1} \leq f$ and $1 \leq l_{2} \leq g$. Clearly the identifiability or the solvability of $\left\{\alpha_{k}, \omega_{k}, \tau_{k}\right\}$ implies that the expression (5.1) of the vector $\left(\beta_{1}, \ldots, \beta_{f}, \gamma_{1}, \ldots, \gamma_{g}\right)^{T}$ for $(3.4-3.5)$ is unique. Thus, to prove the necessity in Theorem 2, we need to prove that the constraint $\left|I_{l_{1}} \cap I_{l_{2}}\right| \leq 1$ and the uniqueness of the expression (5.1) for (3.4 - 3.5) implies the condition in Theorem 2.

Suppose the condition in Theorem 2 does not hold. In other words, there are three distinct integers $k, k_{1}, k_{2}$ such that $\omega_{k}=\omega_{k_{1}}$ and $\tau_{k}=\tau_{k_{2}}$. In the following, we prove that the expression (5.1) for (3.4 $3.5)$ is not unique. Without loss of generality, we assume $k<k_{1}<k_{2}$. Then there exists the following submatrix $A_{0}$ in the matrix $A$ :

$$
A_{0}=\left[\begin{array}{lll}
0 & 0 & 1 \\
1 & 1 & 0 \\
1 & 0 & 1 \\
0 & 1 & 0
\end{array}\right] .
$$

Consider the subsystem of (5.1):

$$
A_{0}\left[\begin{array}{c}
\alpha_{k} \\
\alpha_{k_{1}} \\
\alpha_{k_{2}}
\end{array}\right]=\left[\begin{array}{c}
\beta_{f_{1}} \\
\beta_{f_{2}} \\
\gamma_{g_{1}} \\
\gamma_{g_{2}}
\end{array}\right] .
$$

Consider 


$$
U=\left[\begin{array}{ccc}
1 & 0 & -1 \\
0 & 0 & 1 \\
0 & 1 & 1
\end{array}\right] \quad \text { and } \quad U^{-1}=\left[\begin{array}{ccc}
1 & 1 & 0 \\
0 & -1 & 1 \\
0 & 1 & 0
\end{array}\right]
$$

Let

$$
\bar{A}_{0}=A_{0} U=\left[\begin{array}{lll}
0 & 1 & 1 \\
1 & 0 & 0 \\
1 & 1 & 0 \\
0 & 0 & 1
\end{array}\right]
$$

and

$$
\left[\begin{array}{c}
\bar{\alpha}_{k} \\
\bar{\alpha}_{k_{1}} \\
\bar{\alpha}_{k_{2}}
\end{array}\right]=U^{-1}\left[\begin{array}{c}
\alpha_{k} \\
\alpha_{k_{1}} \\
\alpha_{k_{2}}
\end{array}\right]
$$

Then,

$$
\bar{A}_{0}\left[\begin{array}{c}
\bar{\alpha}_{k} \\
\bar{\alpha}_{k_{1}} \\
\bar{\alpha}_{k_{2}}
\end{array}\right]=A_{0}\left[\begin{array}{c}
\alpha_{k} \\
\alpha_{k_{1}} \\
\alpha_{k_{2}}
\end{array}\right]=\left[\begin{array}{c}
\beta_{f_{1}} \\
\beta_{f_{2}} \\
\gamma_{g_{1}} \\
\gamma_{g_{2}}
\end{array}\right]
$$

where $\omega_{k_{1}}=\omega_{k_{2}}$ and $\tau_{k}=\tau_{k_{2}}$. This gives a second distinct expression of (5.1) for $(3.4-3.5)$. Thus, the necessity is proved.

Since all pairs $\left(\omega_{k}, \tau_{k}\right)$ for $1 \leq k \leq N_{p}$ are distinct, each product set $I_{l_{1}} \times J_{l_{2}}$ has at most one pair. Since $I_{l}, 1 \leq l \leq N_{p}$, and $J_{l}, 1 \leq l \leq N_{p}$, are two partitions of $\mathcal{I}$, it is clear that

$$
\cup_{k=1}^{N_{p}}\left\{\left(\omega_{k}, \tau_{k}\right)\right\} \subset \cup_{l_{1}=1}^{f} \cup_{l_{2}=1}^{g} I_{l_{1}} \times J_{l_{2}} .
$$




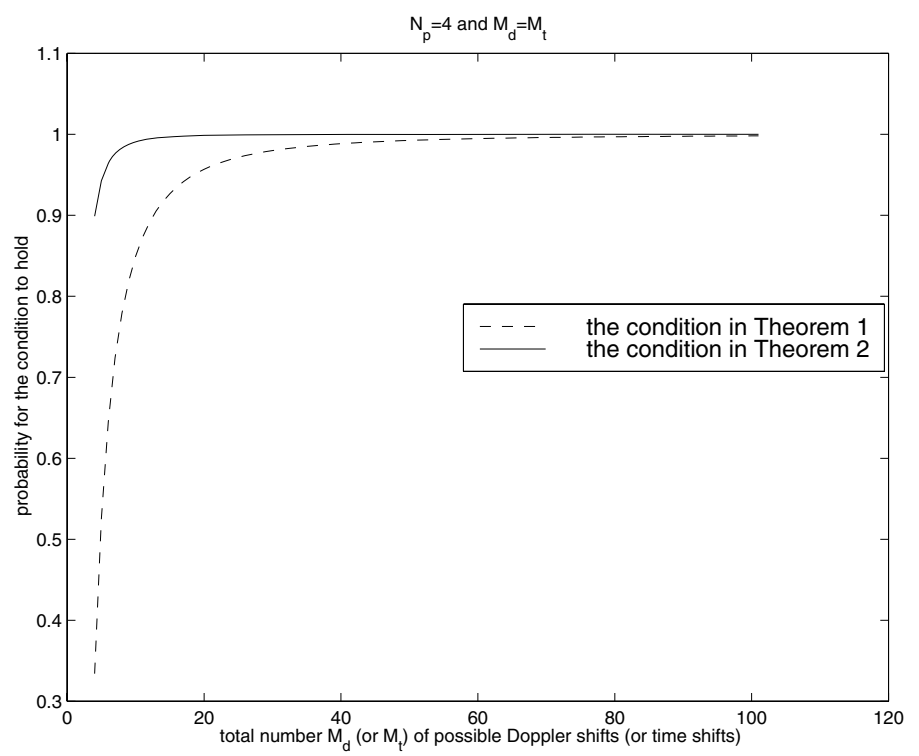

FIGURE 1. Probabilities for the conditions in Theorem 1-2 to hold when $N_{p}=4$.

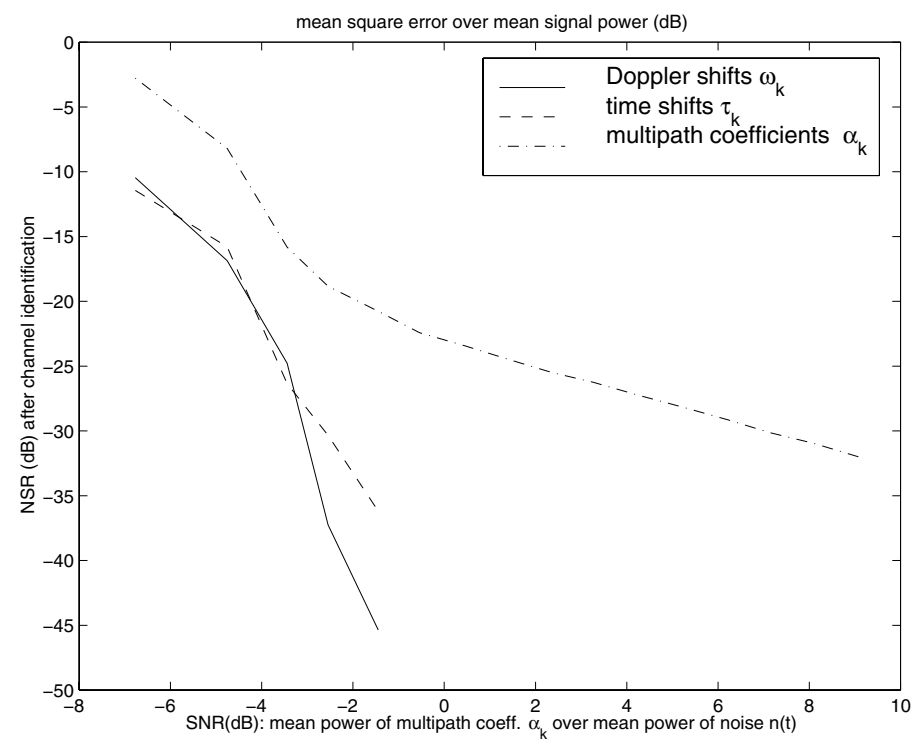

FIGURE 2. MSE for the detected $\omega_{k}, \tau_{k}$ vs. the SNR. 


\section{REFERENCES}

1. M. D. Hahm, Z. I. Mitrovski and E. L. Titlebaum, Deconvolution in the presence of Doppler with application to specular multipath parameter estimation, IEEE Trans. on Signal Processing 45 (1997), 2203-2219.

2. M. D. Hahm and E. L. Titlebaum, Multipath parameter estimation in the presence of Doppler by the method of projection onto convex sets, Proc. CISS (1994), Princeton, 903-908.

3. M. D. Hahm, E. L. Titlebaum and Z. I. Mitrovski, Inverse filtering in the presence of Doppler with application to specular multipath parameter estimation, Proc. ICASSP (1995), Detroit, Michigan, 3167-3170.

4. Z. Kostic, Spread-spectrum coding, performance evaluation and dispersive channel estimation for several classes of code-division multiple-access communication systems, Ph.D. dissertation, Univ. Rochester, 1990.

5. M. Stojanovic, J. Catipovic and J. G. Proakis, Adaptive multichannel combining and equalization for underwater acoustic communications, Journal of Acoustical Society of America (1993), 1621-1631.

6. B. C. Ng, S. N. Diggavi and A. Paulra, Joint Structured Channel and Data Estimation Over Time-Varying Channels, Proc. IEEE/IEICE Global Telecommunications Conference (1997), 409-503.

7. X.-G. Xia, Channel identification with Doppler and time shifts using mixed training signals, Proc. of IEEE ICASSP, Seattle, May 12-15, 1998.

8. T. Zemen and C. F. Mecklenbrauker, Time-variant channel estimation using discrete prolate spheroidal sequences, IEEE Trans. Signal Processing 53 (2005), 3597-3607.

9. J. S. Joo, Channel identification with linear time-varying channel model for pilot channel aided DS/CDMA systems, IEICE Trans. on Communications E89-B (2006), 2652-2654.

10. M. Niedzwiecki, Identification of Time-Varying Processes, John Wiley \& Sons, 2000

Department of Electrical and Computer Engineering, University of Delaware, Newark, Delaware 19716

Email address: xxia@ee.udel.edu 